\title{
Perspective - The long journey to rehabilitation for torture survivors ${ }^{i}$
}

\author{
Kolbassia Haoussou*
}

My name is Kolbassia and I am a co-founder and the Co-ordinator of Survivors Speak OUT (SSO), a UK-based network of torture survivors. My work arises out of my life experiences. First, let me tell you my story.

One evening, when I was just seven, I witnessed my father being persecuted. $\mathrm{He}$ escaped by jumping our wall and running for his life. As a result, we left our home and I had to live in a refugee camp for several years with my grandmother and my younger brother. Later, when I was in my mid-twenties, I myself was tortured.

Like my father, I was forced to flee my home and had to focus on saving myself. My life had crashed around me. I was depressed, had lost my sense of direction and lost the taste and will to live. I thought I would never turn my life around and wanted to end all the suffering by taking my life.

When I eventually arrived in the UK, I was deeply traumatised. I honestly did not know who I was. I isolated myself and I could not trust anyone. I didn't see myself as having a mental health issue. I couldn't. I was from a culture where there is a lot of stigma and shame attached to mental health problems.

After I had been released from immigration detention, a supportive refugee organisa-

*) Co-founder and current Co-ordinator of Survivors Speak OUT, a UK based network of torture survivors, who have been clients of Freedom from Torture. Correspondence to: sso@freedomfromtorture.org tion urged me to seek help to address the symptoms of Post-Traumatic Stress Disorder (PTSD) that I was displaying. I was adamant that I would not be seen by anyone. However, with their support, I eventually became a client at Freedom from Torture $\mathrm{e}^{\mathrm{ii}}$ - and was very grateful that I did.

Freedom from Torture helped me see my experience from a different angle. They gave me a key to turn my life around, but this was only the beginning of my long journey to rehabilitation.

As that journey continued, I asked myself: What is the meaning of rehabilitation for torture survivors like me? And what should we expect of ourselves once formal rehabilitation, for example therapy, ends?

I wanted to explore the issue more thoroughly, to try to develop an understanding of the concept of rehabilitation from the perspectives of survivors, to gain a greater depth of understanding and insight into what they want from rehabilitation and the

\footnotetext{
i This article builds on a presentation given at the International Rehabilitation Council for Torture Victims (IRCT) symposium in Mexico City in December 2016. The symposium theme was 'Delivering on the promise of the right to rehabilitation' and was attended by representatives from torture rehabilitation centres across the world. ii Freedom from Torture is a UK human rights charity dedicated to the treatment and support of torture survivors who seek refuge in the UK through offering psychological therapies and also forensic documentation of torture by expert doctors, practical legal and welfare support, and creative groups.
} 
barriers survivors face in their efforts to overcome their trauma. I also wanted to know whether there could be an end to rehabilitation or whether it is a lifelong quest.

To accomplish these objectives, I had to draw on the experiences of other torture survivors. I did not start with a pre-determined thesis, nor assume that the responses would reflect a common narrative. I determined to listen with an open mind, to understand different perspectives and then see what patterns, if any, emerged.

To put the issue on a more formal footing, together with a researcher from Freedom from Torture, I held focus groups at Freedom for Torture centres, safe spaces where torture survivors could speak of their experiences (information on the methodology we used is listed at the end of this article). Over six months in 2016, we spoke with over 100 survivors of torture at our five centres. We also spoke with staff.

Together we explored two main questions: -What does rehabilitation mean to you? - Does rehabilitation have an end point?

\section{What do survivors want from rehabilitation?}

There were common themes that emerged in almost all our discussions - the need for survivors to move on with their lives while holding on to something of the life they left behind; the desire for independence and the barriers that prevented this; and the need to be empowered and confident to go forward into the future. What emerged was that for rehabilitation to be meaningful, a holistic approach is needed that includes a broad range of considerations.

\section{Moving on or moving back?}

There was a consensus that rehabilitation was about getting survivors back to something like a normal life. How this was defined, and whether this is completely possible, was a key component of our discussions.

"Moving on" was seen as essential to rehabilitation but it means different things for different people. For some people it is a part of the personal journey to "being yourself" so that you could move on by learning "how to forgive, how to forget, how to move on with your life without having flashbacks or memories that make you depressed."

Others felt rehabilitation means having a life that is similar to the life they had back home. They want to experience again some of the things that made them happy before they were tortured, even if they couldn't have exactly the same life they had had before. Some felt this was about helping them return to the person they were before they were tortured, getting their life back.

One survivor said: "We need to think of our former life, the life we had back at home, where we were doing very well, we were training, we were having vehicles, we were having festivities, but having arrived here we have lost everything, we don't have anything. So rehabilitation means for me to have the life which I enjoy back home, at least to some extent."

Some survivors stressed the importance of acknowledging and accepting what had happened to them as a key part of rehabilitation. One said: “Maybe I've spoken to one or two persons, but I'm not ready to say those things again... and if those things are inside you, you cannot move on."

There was a lot of discussion about how survivors could be helped to start a new life as part of the rehabilitation process. Some said that gaining freedom and their rights was crucial. One survivor explained what this means for him: "You have the freedom to do your own stuff, you might want to study or work, but you have that freedom and flexibility to choose what you want to do with your life." 
Other survivors focused on starting a new life as part of the rehabilitation process, they wanted to be able to forget what they had been through and move on.

\section{Independence and restrictions}

Gaining independence and making autonomous choices were widely seen as vital steps on the road to rehabilitation. This included the ability "to stand on your own feet and see a positive future for yourself."

Many survivors said that in order to be truly rehabilitated, their life outside Freedom from Torture has to be stable and secure. Many face significant difficulties as a survivor of torture, and as a refugee or as an asylum seeker, in the UK, and this undermines both their own efforts and the work the organisation does to help them return to normality. Some felt that to be rehabilitated, they needed to be able to make choices about what kind of life they wanted to lead.

A common theme was the legal and societal barriers to independence. Survivors said that until these are removed, they are unable to move on, mentally or physically.

The most commonly discussed barrier was the precarious legal situation that torture survivors experience, with many spending years trapped in the UK's asylum system not knowing whether they will be granted asylum or leave to remain, and the consequent stress and anxiety this creates. One survivor described their situation as: "like a movie when you press pause, you're not moving forward, you're just standing there and waiting for the Home Office."

Almost all the survivors brought up the actions of the Home Office and the problems this creates for them in their everyday lives. Many saw these as a barrier to their rehabilitation. One explained: "I do see benefits by coming here but I'm not in a position to think of the rehabilitation, because I see my life is being destroyed, it is deteriorating, so how can I focus on rehabilitation at this juncture?"

Several survivors felt the actions of the Home Office continually undermined their rehabilitation work with Freedom from Torture. One said: "Because of course here [Freedom from Torture] they're trying to boost our morale and restore everything that we lost, but on the other side the Home Office is pushing us down."

There was a feeling that the Home Office does not act fairly and draws out the asylum process deliberately in the hope that people will give up and return home. Some survivors described this as a form of mental torture. "Back home we are tortured physically but what I've noticed is that the system of the Home Office in this country, they are trying to torture us mentally, and I think it is worse than the physical torture that I've been through."

Achieving freedom from the restrictions currently placed on them is an important part of rehabilitation for a number of survivors. This includes being allowed to move freely within the country, having access to employment, getting a driving license, and even simply being listened to by the authorities.

This was especially true for those with children who felt unable to provide for their children properly while restricted in this way. "As far as children are concerned, the money that we are getting is not sufficient enough to meet their needs. What did they do to have such a lifestyle, whilst others are enjoying a different lifestyle?'

Other issues described by survivors as barriers to rehabilitation included finding work, when they finally have legal status to remain in the UK and permission to work, and the difficulties of learning a new language. One survivor explained: "In my 
country I don't speak English, you need to learn English because everything here is in English, you need to learn more. Here I need to start a new life, a new language, new people. It's not easy to start a new life. Not the life I had before."

Survivors also face more nebulous barriers. These include not understanding the (often unwritten) rules of society and difficulties assimilating into the new culture. One survivor said: "The big problem is we can't make ourselves understood and they can't understand us, where we're coming from and why we are asking for certain things, it's cultural issues."

In addition, society lacked knowledge of the needs of survivors of torture. "They just don't know; they just don't understand why you're suffering with something you experienced twenty years ago."

Some survivors said that the profound effects of torture makes rehabilitation even more difficult, especially when it comes to difficult tasks involving retaining new or complex information or learning a new language. "[Because of] the torture I received, the attention of my mind is stopping learning the language because no matter what I do I just can't retain.” Another said: "As you can see I'm physically disabled. This is not coming from nowhere, this is not a natural disability, this is from torture. But I try to put all this behind me because of the help of the therapy."

A number of survivors also spoke of the negative impact of discrimination. "It is very hard to reach a point [of rehabilitation] when you get people dealing with you in this disrespectful way. It has a knock-on effect on your morale, on your confidence, on your ability to be positive and work and deal with the struggles in your situation." One survivor felt so threatened in the area where he lived that he was unable to attend therapy sessions, or even leave the house.

\section{Empowerment and confidence}

Feeling confident and empowered was seen as a key aspect of the rehabilitation process. Participants talked about being empowered to take back control of their life and meaningfully navigate their own circumstances.

Several survivors spoke of the positive effect of feeling productive, and the possibility of restarting the career they had before they were tortured. Many people suggested that access to courses, training and careers advisors could help them to grow in confidence and become active members of society. One person said that it is important to "bring out the best in other people... because a lot of us, we've got different talents." Survivors said they sought opportunities to rebuild their confidence, either through training or through becoming active in the communities where they live.

Others talked of the importance of being busy, socialising and not spending all of their time isolated in their house. This was a particular concern for those housed by the Home Office in more remote, sometimes hostile, areas. Activities which aided people's confidence and alleviated their isolation included going to the gym, swimming and volunteering. One survivor suggested: "when you keep your mind occupied by doing something, get busy, you know it helps keep your mind out of things."

Being able to speak out about what happened to them, and advocating for other survivors with decision makers or to public audiences is a key part of rehabilitation for some survivors. They said that using their voice empowers them and helps restore their confidence. This is especially important for people who previously felt unable to use their voice to speak out in public.

However, there are barriers that many find hard to overcome. Several survivors expressed the view that torture, and their 
experiences in the UK asylum system, had drained their confidence. "You know when you are in one stage of life for a very long time it brings your confidence down, right down, so you need something that will motivate you that will help you to get back that confidence."

\section{Can there ever be an end to rehabilitation?}

All the survivors agreed that rehabilitation is a very long process, with no short cuts. One said: "All the nightmares and the memories and the struggle I've had over the last twenty years is not going to go away with three or six or twelve months of therapy here; it's an ongoing thing."

Several survivors described the deep impact torture had had on their lives: "What we experience under torture, it changes you from inside [which means] it is an ongoing situation that needs monitoring and looking after." Many spoke about how long, difficult and uncertain rehabilitation could be. They spoke about being uncertain of how fully they were recovering, as their mood and feelings changed from day to day.

While all the survivors stressed that rehabilitation could not happen without secure legal status, they also said that, in itself, legal status did not end their stress and anxiety. They would find themselves moving on to a new stage of their life with new difficulties, and the effects of their trauma would not simply disappear. "You still have nightmares because the papers [immigration documents confirming legal status] don't stop your nightmares. You still weep and walk; it is not that papers give you the full joy."

In fact, gaining legal status and permission to remain in the UK could bring new problems, at least in the short term. These include losing the (minimal) income and housing available to people seeking asylum, looking for work and finding new housing, all within a short time frame. One survivor said:
"The added problems, even after your status is granted, just pour in."

The ongoing uncertainties of life and the barriers survivors face made many feel that, if there is going to be an end point to their rehabilitation, it could not be achieved without feeling secure about their accommodation, their income, and their legal status.

Some survivors felt that, for them, rehabilitation would never be achieved and that the effects of torture will remain with them for their rest of their lives. They said this is partly because, even if they are improving, they feel that stresses in life can always bring back their past trauma. "The added problem, it [life stress] reignites the problem I have, the nightmares and the depression."

A number of survivors also stressed that sometimes other people assume that a person is fully rehabilitated when they do not feel that they are. "People think, oh you have moved on, but within you still know you are just going round in a circle.'

Some survivors felt they would always need therapy to cope with the continuing stress. "I think it's not something that should end, it's an ongoing thing that should go on and on." Some said that it is always possible they would have a setback. "It is not a physical illness where you take a medication and you're sorted, it is an ongoing situation that needs monitoring and looking after."

Others felt that they would always be working to engage with life, so rehabilitation would be an ongoing process throughout their life. One client compared it to having a hand cut off. "You won't grow a new hand but you'll learn how to adapt and that takes a lifetime."

But not everyone believed that rehabilitation would be lifelong. Some survivors said that once they reach a point where they are feeling better, working and living a normal 
life, their rehabilitation would end. The trauma would remain, but it would not affect them in the way it had in the past - they would learn how to deal with it.

\section{The importance of therapy}

All the survivors we spoke with had been offered therapy at Freedom from Torture. I found that for many torture survivors, rehabilitation as we know it in western countries, was unknown and unfamiliar; talking to a therapist individually or in a group was unchartered territory. Like me, many people had preconceived, negative views of mental health, which was associated with stigma and shame.

Although the concept of therapy was foreign to most, they all came to value it highly. All said that physical and psychological therapy, both in one-to-one and in group settings, was a very important part of their rehabilitation.

First, it meant they had someone to listen to them. One survivor said: "We are here alone and the only hope we have is at least someone to listen to us." Another said: "Freedom from Torture was the only organisation that actually took time to sit down with us and look at the whole picture."

Second, therapy has enabled them to start to address their trauma in ways that are constructive and has helped them deal with the terrors they face, such as intrusive memories, flashbacks and nightmares.

Survivors spoke of the difficulty of dealing with the effects of the torture they had experienced while also dealing with new problems: 'I deal with two problems, whereas most people deal with one... I have to go back and get rid of the traumas in the past as well as the problem today."

They said that therapy has given them more resources to cope with day-to-day life and the many ongoing difficulties they face.
"The services you [Freedom from Torture] provide are of great help to me, and the treatment I have received from you. Without it, it would be a lot harder."

One-to-one therapy was not the only method of rehabilitation that was valued. Many survivors spoke highly of the range of other activities that are provided by Freedom from Torture, which brought them together with other survivors and helped overcome isolation. This includes activities such as football, gardening and cooking, and the service users group (which enables current and former service users help shape Freedom from Torture's services).

These activities were seen as particularly helpful as they allow clients to keep occupied and engage with people who are going through similar experiences: "It is good to go in a group to see that you're not the only person struggling. There are other people like you."

Attending meetings with other torture survivors was also seen to be an important part of rehabilitation. These help survivors deal both with their current problems and their past trauma: "When we come together to discuss, it is helpful, because when I am alone it is not easy to deal with those things."

However, for some survivors the meetings are bittersweet as talking together with others reminds them of what they have gone through, and the life they had before they were tortured. The shared memories of home, the knowledge of shared customs, culture and lifestyle, can bring comfort and reassurance, together with sadness and homesickness. One survivor expressed his feelings in this way: "When we talk about these things, I am reminded of what life I had back home, so in a way I would like to avoid coming for this meeting. But on the other hand there's a hope when I come here that we will do some sort of help so that we can have a life, which we are longing for." 
Some survivors also spoke about their involvement in making a contribution to the wider work of Freedom from Torture through service expert panels and how this contributes to their rehabilitation. One said: "We feel like we were part of it, and when we go home we feel better, so it is like part of therapy." For these current service-users and for former service-users who are now members of the Survivors Speak OUT network, having their concerns listened to and acted upon, and then, if they chose to, becoming an advocate in their own right, is a part of their rehabilitation process.

Others suggested that they wanted to help Freedom from Torture grow so it could reach other survivors who would benefit from its services: "It makes me also feel better about myself, that I am doing something back for the society."

Other survivors also expressed support for the work Freedom from Torture does to influence policy and decision makers, to try to improve the asylum system so it works more humanely and effectively.

Some survivors said that legal and welfare advice services contribute significantly to their rehabilitation, by helping them to address many of the practical and legal difficulties that arise during the asylum application process and beyond. Survivors said that it makes a big difference to them if they feel supported and not alone in their dealings with the Home Office. Survivors suggested that without such attention, these problems would continue to act as barriers to rehabilitation. One said: 'I can say that there's no way that we can call it rehabilitation, or we can do the rehabilitation process if for example the Home Office is there just to let us down."

\section{Common themes - Lessons learnt}

Despite the varied experiences and responses revealed in the discussions, a few common themes emerged. The first is that torture is extremely damaging to physical and mental health and torture survivors want and need humane, consistent and tailored rehabilitation support before and during the asylum process and after they have gained refugee protection. For some survivors this is a lifelong process. Others learn better how to cope with their trauma, although many fear that the damage from their past torture can always return in unforeseen and unexpected ways.

A second theme is that the dysfunctions and delays in the asylum system play a huge role in exacerbating the trauma of torture. Individuals who are trapped in the asylum system for months and years, often going through multiple tribunal hearings, their experiences being routinely disbelieved by caseworkers, find their lives on hold, unable to move forward.

Added to that are the privations of living on meagre benefits, being accommodated in inadequate housing, often with strangers in a hostile area, and the indignities of being unable to work or study. All these factors hinder effects at rehabilitation.

A third theme is that therapy can be effective in helping torture survivors on the road to rehabilitation. Although rehabilitation through psychotherapy is an unfamiliar concept to many, survivors feel that it helps them to make sense of their own experiences. Just being listened to and being seen as a whole person is seen as being of value in itself. Group therapy that enables survivors to be active, to meet and connect with each other plays a very positive role in rehabilitation.

\section{My journey to rehabilitation}

I have been reflecting on some of my own experiences which happened over ten years ago, but still my rehabilitation journey 
continues. Throughout that journey, I have often wondered when rehabilitation begins and when it ends - or indeed whether there is an end. You might be surprised to hear that I strongly believe that my journey to rehabilitation started, not during therapy, but when I was taken from my home that evening and tortured.

Looking back to various moments of my own experience of rehabilitation, I felt different things at different times. Sometimes I wished I could have become the old person I used to be before torture. And sometimes I would have liked to be a different version of my old self - more prudent and far-seeing but still rebellious against torture. At other times, I would have liked just to disappear somewhere, build a quiet life and forget everything.

But, again, my anger at the treatment I received in my home country and then later in the UK through the asylum process wouldn't allow me to forget and be quiet. That was the anger that drove me to become a co-founder of Survivors Speak OUT, a network of torture survivors who speak out against torture and its impact on our lives.

I see rehabilitation as a soul-searching journey of pain, joy, acceptance of the past and perhaps the present, but also rediscovering. Above all, it is also about finding resilience and strength to accept yourself in all that you have been through and all that you now are or will be. It is a continuous journey.

Through the survivors I spoke to about the meaning of rehabilitation, I learnt that this is about holistic support using conventional approaches to rehabilitation through therapy but that it is also about feeling empowered, finding independence in the host country, securing protection without a risk of return to torture, and ultimately regaining the confidence that many of us lost.
One thing that torture attempts to achieve is to take away the ownership of your power and your voice. Torture reduces you to silence; you feel numb. That is why it was crucial for me and for us survivors to speak up so that we will never be silenced, and so that, as a group of survivors, we can advocate for other victims and survivors. For us, that is also an important part of rehabilitation.

\section{Acknowledgements}

I wish to thank all the survivors of torture who participated and gave so generously of their time and experiences.

\section{Afternote}

The research referred to in this article was designed in collaboration with the research department at Freedom from Torture. A qualitative design was adopted, in order to explore the range and diversity of views and experiences of survivors of torture on the topic of rehabilitation. It was decided that focus groups would be the most appropriate method, as the group setting would enable participants to share their experiences and would allow for a rich discussion based on a range of views and perspectives. Focus groups would also be less time intensive than individual semi-structured interviews and would enable a larger number of people to participate.

Participants were recruited from existing service-user groups in each of Freedom from Torture's five centres in London, Birmingham, Manchester, Glasgow and Newcastle. Service-users who are active in these groups are comfortable sharing their views and discussing sensitive issues in a group setting and the majority therefore consented to participate in the research. Although participants were recruited using an 'opportunistic' approach, there was a reasonably good overall level of diversity in the groups in 
terms of: nationality of origin, gender (although the majority were male), age and length of time that participants had been in receipt of Freedom from Torture's rehabilitation services. Participants also included those who are currently awaiting the determination of their asylum claim, and those who had been granted refugee status.

The research was conducted over a period of six months. Focus groups lasting between 90-120 minutes were co-facilitated by Freedom from Torture's researcher, Jo Pettitt, and the survivors advocate and Survivors Speak OUT network co-ordinator, Kolbassia Haoussou. Over 100 participants were involved in the research overall. In the London centre, which has the largest number of service-users, three parallel focus groups were held, with one focus group in each of the other centres. An additional focus group was held with Freedom from Torture staff in the London centre.

To enable meaningful participation, time was spent understanding the purpose of the research and terminology. Interpreters also attended, where needed. Focus groups were audio recorded with the permission of participants, and each was transcribed verbatim.

The findings were analysed systematically using thematic analysis, which is an appropriate method for identifying, analysing and reporting patterns or themes arising from research data. This approach allows for data led findings to emerge, rather than imposing a theory onto the data. This was considered appropriate given the exploratory nature of this research. 